sive Bande bei $2,747 \mu$ besitzt. Die Verstärkung der Ultrarotabsorption des $2-0 x y-2^{\prime}, x^{\prime}$-dinitrostilbens im benachbarten $\mathrm{CH}$-Gebiet weist offensichtlich auf eine Verlagerung dieser fehlenden OH-Absorption in das Gebiet der CH-Valenzschwingungen hin, wie sie bei Sechsringen bekannt ist ${ }^{1}$ ).

Die Analogie zum chemischen, ultrarotspektroskopischen und Adsorptionsverhalten ${ }^{2}$ ) der bekannten

I) Buswell, Deitz und Rodebush, Journ. Chem. Phys. 5, 501 (1937).

2) H. Hoyer, Kolloid-Z. 116, 121 (1950); Z. Elektrochem., angew. physik. Chem. 54, 413 (1950).
Wasserstoffbrückensechsringe ist vollkommen. Damit ist bewiesen, daß es zehngliedrige Ringe mit starker innermolekularer Wasserstoffbrücke gibt. Durch die Auffindung dieser Zehnringe bei Stilbenderivaten eröffnet sich die Aussicht, in der Lage der OH-Banden ebener und unebener Isomere (trans- und cis-Formen) ein Kriterium für die Beteiligung der Resonanz beim Zustandekommen der Wasserstoffbrückenbindung zu gewinnen.

Herrn Prof. Dr. Mecke danke ich für die Aufnahme der Ultrarotspektren, Herrn Dipl.-Chem. Corcilius für Mitarbeit bei der Präparation.

\title{
Berichtigung
}

\section{Einige Beispiele für organische, mit Wirkungsgruppen ausgerüstete Hochpolymere und Permutoide : ihre Herstellung, ihr optisches und reaktives Verhalten}

Von W. La utsch unter Mitarbeit von W. Broser, W. Biedermann, U. Döring und H. Zoschke Kolloid-Z. 125, 72 (1952)

Auf Seite 73 muß es heißen:

„Die Aufnahme der Spektren des obenerwähnten Chlorin- $e_{6}$-dimethylester-6-säure-äthylamids bzw. - polyäthylenimids bei verschiedenem $\mathrm{p}_{\mathrm{H}}$ ermöglichte die Zuordnung der verschiedenen Spektraltypen $\mathrm{zu}$ denen der freien Base, des einfach und doppelt geladenen Kations, sowie die Ermittlung der Dissoziationskonstanten der Kationensäuren beider Verbindungen, deren negative Logarithmen die $p_{K}-$ Werte darstellen ${ }^{3}$ ). Es ergaben sich bemerkenswerte Unterschiede bei der niedermolekularen Verbindung und derselben als im Hochpolymeren gebundenen Wirkungsgruppe: Die $\mathrm{p}_{\mathrm{K}}$-Werte der einfach geladenen Kationensäure betrugen im ersteren Falle 2,7; im letzteren 3,75.“

\section{Bücherbesprechungen}

Gmelins Handbuch der anorganischen Chemie, 8. Auflage, herausgegeben vom Gmelin-Institut in Clausthal-Zellerfeld. (Weinheim 1950, Verlag Chemie).

Dem Berichterstatter liegen vor: System Nr. 18, Antimon, Teil A, Lieferung 3 (1950), Bildung und Darstellung des Metalls; Nr. 28, Calcium, Teil A, Lfrg. I (1950), Geschichtliches; Nr. 35 Aluminium, Teil A, Lfrg. 8 (1950), Ternäre Legierungssysteme $\mathrm{Al} / \mathrm{Fe} / \mathrm{C}$ und $\mathrm{A1} / \mathrm{Fe} / \mathrm{Si}$; Nr. 62, Gold, Lfrg. 1 (1950), Geschichtliches; Nr. 68, Platin, Teil A, Lfrg. 6 (1951), Die Legierungen der Platinmetalle Osmium, Iridium, Platin; Nr. 41 Titan (1951) und die einleitenden Seiten VI-XXVII.

Es erübrigt sich, an dieser Stelle auf die Bedeutung der achten Auflage des Gmelin hinzuweisen. Ebenso allgemein bekannt dürfte es sein, da $B$ die Herausgeber erst in jahrelanger Arbeit die fast völlige Zerstörung des Archivs und aller ihrer Hilfsmittel überwinden mußten, bis sie vor etwa zwei Jahren wieder mit den ersten Lieferungen hervortreten konnten. Die neuen Verhältnisse erforderten jedoch eine Änderung des bisherigen Arbeitsplanes. Durch strafferes Zusammenfassen und durch Beschränkung der Grenzgebiete auf das für den Chemiker Notwendige soll der Umfang des Werkes herabgesetzt und der vollständige Abschluß in etwa zehn Jahren ermöglicht werden. Als Stichtag für die Erfassung der Literatur wurde für alle noch erseheinenden Bände einheitlich der 1. Januar 1950 festgesetzt. Ergänzungsbände sollen die bereits erschienenen älteren Teile ebenfalls bis zu dem allgemeinen LiteraturschluBtermin 1. 1. 1950 fortführen. so daß nach ihrem $A b$ schluß die 8. Auflage des Gmelin das gesamte Schrift- tum der anorganischen Chemie und ihrer Randgebietbis Ende 1949 enthalten wird.

Die Neuauflage des Gmelin wurde durch die größtmögliche Vollständigkeit und Zuverlässigkeit der Angaben weltbekannt, und auch die bisher erschienenen Nachkriegslieferungen zeigen dieselbe Sorgfalt der Bearbeitung. Die oben erwähnte Beschränkung des Arbeitsplanes bringt für Interessenten aus den Randgebieten die Gefahr mit sich, daß sie das ihnen wichtige Material im Gmelin nicht mehr so vollständig bearbeitet fïnden wie bisher. So werden die Angaben über Nachweise und Bestimmungsweisen der Elemente in Zukunft dem Handbuch der analytischen Chemie überlassen. Für das Gebiet der Kolloidchemie besteht aber wohl keine Befürchtung; dies zeigen die vorliegenden Teillieferungen. Entsprechend der kulturgeschichtlichen Bedeutung des Goldes werden in der Lieferung 1 ausführliche Angaben über die schon im Altertum bekannte Herstellung von Rubinglas gemacht, ein eigenes Kapitel ist der Geschichte des kolloiden Goldes gewidmet, und auch die Abschnitte über das Färben von Gold werden beim Kolloidchemiker Interesse finden. Auch der neue Titanband widmet nicht nur der Darstellung und den Eigenschaften von Titandioxyd-Solen ein eigenes Kapitel, sondern bringt auch in den andern $\mathrm{Ab}$ schnitten dem Kolloidehemiker zahlreiche Anregungen. Ebenso sei an dieser Stelle auf die Literaturzusammenstellung über kolloides Antimon im Antimonband A 3 hingewiesen, die zahlreichen Einzelangaben von kolloidchemischer Bedeutung, vor allem in der Platin-Teillieferung, sind wohl nur bei intensivem Durcharbeiten 